\title{
FFPE breast tumour blocks provide reliable sources of both germline and malignant DNA for investigation of genetic determinants of individual tumour responses to treatment
}

\author{
Anna Wilkins ${ }^{1,2,4} \cdot$ Ritika Chauhan $^{3} \cdot$ Alistair Rust $^{3}$ - Alex Pearson ${ }^{5}$. Frances Daley ${ }^{5}$. Floriana Manodoro ${ }^{3}$. \\ Kerry Fenwick ${ }^{3}$. Judith Bliss ${ }^{2} \cdot$ John Yarnold ${ }^{1,4} \cdot$ Navita Somaiah $^{1,4}(\mathbb{0}$
}

Received: 5 January 2018 / Accepted: 19 April 2018 / Published online: 26 April 2018

(c) The Author(s) 2018

\begin{abstract}
Background Bio-banked formalin-fixed paraffin-embedded (FFPE) tissues provide an excellent opportunity for translational genomic research. Historically matched blood has not always been collected as a source of germline DNA. This project aimed to establish if normal FFPE breast tissue could be used as an alternative to blood.

Methods Exome sequencing was carried out on matched tumour tissue, normal breast tissue and blood on five patients in the START trial. Retrieved samples had been archived at different centres for at least 13 years. Following tissue macrodissection and DNA extraction, targeted exome capture was performed using SureSelect Human All Exome v5 reagents (Agilent). Illumina paired-end libraries were prepared from the captured target regions and sequenced on a HiSeq2500 (Illumina) acquiring $2 \times 75 \mathrm{bp}$ reads. Somatic variants were called using the MuTect software analysis tool and copy number abnormalities (CNA) were identified using CNVkit. Targeted sequencing and droplet digital PCR were used to validate somatic variants and CNA, respectively.

Results Overlap of somatic variants and CNA called on tumour versus blood and tumour versus normal breast tissue was good. Agreement in somatic variant calling ranged from 76.9 to $93.6 \%$. Variants with an allele frequency lower than $10 \%$ were more difficult to validate irrespective of the type of germline DNA used. Pearson's correlation coefficients for paired comparisons of CNA using blood or normal tissue as reference ranged from 0.70 to 0.94 .

Conclusions There is good correlation between the somatic mutations and CNA called using archived blood or normal breast tissue as germline reference material.
\end{abstract}

Keywords Germline determinants · Whole exome sequencing $\cdot$ Breast cancer · Archival formalin fixed paraffin embedded tissue

Electronic supplementary material The online version of this article (https://doi.org/10.1007/s10549-018-4798-7) contains supplementary material, which is available to authorised users.

Navita Somaiah

navita.somaiah@icr.ac.uk

1 Division of Radiotherapy and Imaging, The Institute of Cancer Research, London, UK

2 The Institute of Cancer Research Clinical Trials and Statistics Unit, London, UK

3 Tumour Profiling Unit, The Institute of Cancer Research, London, UK

4 The Royal Marsden Hospital, Downs Road, Sutton SM2 5PT, UK

5 Division of Breast Cancer Research, The Institute of Cancer Research, London, UK

\section{Introduction}

Bio-banked formalin-fixed and paraffin-embedded (FFPE) tissues provide an excellent opportunity for translational research. Reductions in cost and the wider availability of next-generation sequencing (NGS) in the research community has meant that this sequence-level analysis is increasingly being used in cancer genetics. For DNA studies, matched germline blood is typically used for exome and whole genome sequencing to provide a "normal" reference against which an individual patient's abnormal tumour DNA can be compared. Historical clinical sample collection, prior to the widespread availability of NGS, did not usually include collection of germline blood. An important pragmatic question, therefore, arises as to whether surrounding 
normal tissue can be used as an alternative reference germline from these archival samples. This proof-of-principle study was designed to assess the feasibility and validity of using normal breast tissue as a reference germline in a small cohort of patients where all three of normal breast tissue, breast tumours and blood samples were available in a historical cohort.

"Field cancerisation" describes areas around tumours consisting of histologically normal, yet genetically aberrant cells, and is well-recognised in breast cancer [1]. Genomic alterations including allelic imbalance and loss of heterozygosity (LOH) [2], aneusomy [3] and dysfunctional telomeres [4] have been demonstrated in histopathologically normal breast lobules, and the frequency of some aberrations has shown a correlation with distance from the tumour [5]. Such changes typically occur in only a proportion of epithelial cells and are unlikely to be present in other cell types present in normal breast tissue. Other cell types include adipocytes, stromal, endothelial and smooth muscle cells, and possibly cells associated with inflammation or the immune response. The impact of field cancerisation may therefore be minimised by selection of normal breast tissue located at a distance from the tumour, which will typically include a mixture of epithelial and non-epithelial cells.

A previous study used polymerase chain reaction (PCR) to assess five genotypes with frequent $\mathrm{LOH}$ in breast cancer and demonstrated $100 \%$ concordance for genotyping from FFPE normal tissue adjacent to tumour and from blood [6]. The International cancer genome consortium (ICGC) conducted a large sequencing study to assess the landscape of somatic mutations in 560 breast cancer whole-genome sequences [7]. For 96 patients adjacent breast tissue was used as the source of normal DNA, whereas for 353 patients blood was used as the source of germline DNA. Although a formal comparison between the sources of germline DNA was not reported, no significant differences in sequencing findings were noted in cases using adjacent breast versus those using blood for germline DNA.

The cancer genome atlas (TCGA) recently conducted a multi-platform analysis using fresh frozen samples. DNA defects, epigenetic changes and gene expression profiles were reported from cancer-adjacent breast tissue, defined as at least $2 \mathrm{~cm}$ from breast tumour [8]. Although changes consistent with field cancerisation were identified, these did not appear to prohibit use of normal breast tissue as the germline reference. Sequence data from tumours and cancer-adjacent breast tissue were each compared with blood, and therefore the genomic comparisons conducted in the TCGA analysis were not the same as our study.

The work in this study aimed to establish if DNA extracted from archived normal breast tissue blocks can be used as a surrogate germline reference by comparing exome sequencing of breast tumours using either matched blood DNA or normal breast DNA as the germline reference.

\section{Methods}

\section{Selection of patient samples}

Five patients with contemporaneous plasma and breast samples were selected from different centres participating in the START radiotherapy fractionation trial (ISRCTN59368779), so as to represent a cross section of the larger trial cohort. Matched archival FFPE blocks for tumour tissue and normal breast tissue plus frozen blood samples for these patients were identified. The samples had been stored for at least 14 years. Normal breast tissue blocks were chosen that were considered as free as possible of tumour cell contamination or pre-malignant change, following review of Haematoxylin and Eosin (H \& E) sections by a specialist breast cancer pathologist. This means that individual sections flanking the $\mathrm{H} \& \mathrm{E}$ section were highly likely to be free of malignant or pre-malignant change. Eligibility criteria for the START trial included complete macroscopic excision of tumour by breast-conserving surgery or mastectomy (ideally, no microscopic evidence of invasive or in situ cancer at, or within $1 \mathrm{~mm}$ of, a resection margin). Three patients had undergone lumpectomy plus wide local excision and two patients had undergone mastectomy. It was not possible to retrospectively quantify the exact distance from tumour but the existence of tumour-free blocks suggests the margins from invasive tumour were generous.

\section{DNA extraction}

Sections $(3 \times 8 \mu \mathrm{m})$ were cut from tissue blocks, and stained with Nuclear Fast Red to guide macro-dissection. Tumour areas were macro-dissected following demarcation of malignant tissue on an $\mathrm{H} \& \mathrm{E}$ slide by a specialist breast pathologist, with the aim of maximising tumour purity. Normal breast tissue blocks did not undergo any macro or microdissection. Tumour and normal breast DNA was extracted using the QIamp DNA FFPE Tissue Kit (Qiagen) as per the protocol instructions. DNA was eluted in approximately $70 \mu \mathrm{l}$ of ATE buffer and stored at $-20^{\circ} \mathrm{C}$. DNA from blood was extracted using the Qiagen Blood and Tissue kit following the manufacturer's instructions. DNA was quantified using Qubit fluorometry.

\section{Exome sequencing and data analysis}

Targeted exome capture was performed using SureSelect Human All Exome v5 reagents (Agilent). Illumina pairedend libraries were prepared from the captured target regions 
and sequenced on a HiSeq2500 (Illumina) using v4 chemistry acquiring $2 \times 75$ bp reads.

BWA-mem (v0.7.5a) was used to align reads to the human reference genome (GRCh37). PCR duplicates were removed prior to further processing and variant detection. Somatic variant calling on tumour samples paired with matched normal samples was performed to identify both single nucleotide variants (SNVs) and small insertions/deletions (indels) of $<30 \mathrm{bp}$. Somatic SNVs were identified using MuTect (v1.1.4; http://www.broadinstitute.org/cancer/cga/mutect). Somatic indels were selected using the Genome Analysis Toolkit (GATK) v3.3.0. All somatic variants were annotated using the SnpEff [9], which provided information on genes affected by mutations and the likely consequences for the encoded gene products. Variants called in regions not covered by the capture probes were excluded, as were those with genotype quality scores below 20 , alternative read count less than 10, variant allele frequencies (VAFs) less than $2 \%$ and coverage less than 20 reads in either sample.

CNVKit v0.8.5 [10] was used to derive somatic copy number alterations (CNA) by comparing the tumour sample to the matched germline sample (normal breast tissue or blood). CNVkit calculates the mean read depths for ontarget and off-target reads which are then combined for each interval. The combined mean read depths were normalised against the control sample and corrected for systematic biases (such as GC content) to obtain the $\log 2$ copy number ratios. Discrete copy number segments were generated from the bin level $\log 2$ ratio values using the circular binary segmentation (CBS) algorithm [11], which is available as one of the segmentation methods within CNVKit. The copy number values and frequency of heterozygous mutations were used for the estimation of tumour purity using the ASCAT software package (8). The observed $\log 2$ copy number ratios were adjusted using the purity estimates to generate the absolute copy number values. Adjacent segments with the same allele specific copy number were merged using the "filter cn" option in CNVkit.

Two different approaches for description of CNA were used; matched and pooled. First, individually matched tumour versus normal tissue or blood comparisons was conducted. Second, a pooled analysis using all tumour-free normal breast tissue samples or all blood samples as reference germline, compared to each individual tumour sample, was performed [12].

\section{Validation of somatic variants using a customised AmpliSeq panel}

A customised Ampliseq panel assessing 139 somatic variants detected using blood or normal breast tissue as reference was developed using the Ion AmpliSeq Designer. This panel was designed to validate mutations with a discrepant call between normal breast and blood, and variants of particular relevance to cancer [cancer genomics consortium (CGC) genes]. In order to explore the limit of detection of variants, it also included a number of variants with a VAF on exome sequencing that was lower than $10 \%$. As there were slightly more somatic variants detected using blood as germline versus normal breast, the Ampliseq panel was enriched for discrepant variants detected using blood as the germline $(n=35)$ rather than normal breast as the germline $(n=4)$.

Sequencing libraries were prepared using the automated Ion Chef library preparation protocol, using a single pool of $10 \mathrm{ng}$ of DNA. Libraries were templated using the Ion Chef (Life Technologies), and sequenced on a PI chip using the Ion PI HiQ sequencing reagents (Life Technologies), 520 flows, with an average amplicon length of $117 \mathrm{bp}$, to a minimum depth of $\times 18,000$. The sequencing resulted in $3,363,175-7,408,316$ reads per sample. The sequencing data were processed using the Ion torrent suite v5.0.4. Reads were aligned to the human reference genome (GRCh37). Coverage data were generated using the Coverage Analysis plugin v5.2. Ion torrent Variant caller (TVC) plugin v5.2 with no Hotspot region and the configuration "Somatic Low Stringency" was used for calling variants. The variants called in the Ampliseq panel were then compared with the Illumina exome sequencing data.

\section{Validation of copy number abnormalities using digital polymerase chain reaction (ddPCR)}

Droplet digital PCR (ddPCR) was used to validate copy number abnormalities. ddPCR was performed on a QX200 digital PCR system (Bio-Rad) using the assays described in table $\mathrm{S} 1$. CGC genes with the highest copy number gain ( $\geq 4$ absolute copies) were prioritised as these are likely to be of most clinical impact and ddPCR has been shown to detect these robustly $[13,14]$. Genes showing different copy number gain using either blood or normal breast as a reference were also prioritised to indicate which reference germline provided most accurate estimation of the relevant CNA. 1-2 ng of tumour tissue DNA was used per assay. Reactions were prepared using digital PCR Supermix for probes (Bio-Rad) and a QX200 droplet generator, this system produces approximately 20,000 droplets per reaction. PCR experiments were run on a 96-well plate on a G-storm GS4 thermal cycler, incubating the plates at $95^{\circ}$ for $10 \mathrm{~min}$ followed by 40 cycles of $95^{\circ}$ for $15 \mathrm{~s}$ and $60^{\circ}$ for $60 \mathrm{~s}$, then $10 \mathrm{~min}$ incubation at $98^{\circ}$. Plates were read on a Bio-Rad QX200 droplet reader with QuantaSoft v1.6.6.0320 software to quantify the number of droplets positive for the genes being assessed. Four reference genes were used for each test gene, together with at least two negative control wells with no DNA in every run. Positive 
and negative controls using cell lines with and without the relevant copy number alteration, respectively, were also included in each experiment. CNA for target genes were calculated as a ratio with the median of the four multiplexed reference genes.

\section{Results}

\section{Quality of NGS}

Sample description, library size, exome capture and sequencing metrics are displayed in Table 1. Overall, these indicate that the DNA libraries were of good quality and exome capture was performed robustly with more than $100 \mathrm{X}$ median coverage for all samples. Exome capture and coverage was

Table 1 Sequencing quality control

\begin{tabular}{lll}
\hline Sequencing parameter & FFPE samples $(n=10)$ & Blood $(n=5)$ \\
\hline Library size (bp) & $236-278$ & $324-328$ \\
Exome capture \% mapped & $89-94 \%$ & $96 \%$ \\
\% Duplicates & $10-15 \%$ & $6 \%$ \\
\% On targets & $74-78 \%$ & $70-74 \%$ \\
Median depth & $134 \mathrm{X}-167 \mathrm{X}$ & $107 \mathrm{X}-125 \mathrm{X}$ \\
\hline
\end{tabular}

FFPE formalin-fixed paraffin-embedded

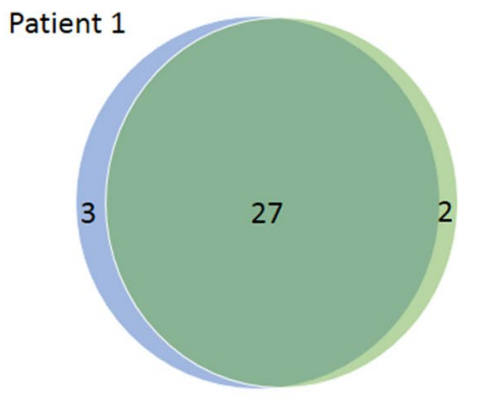

Patient 3

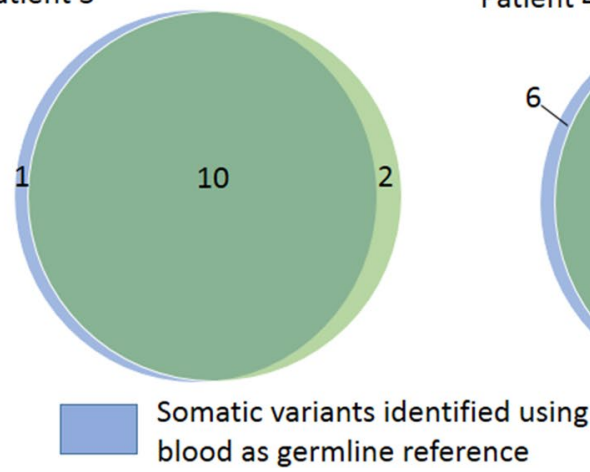

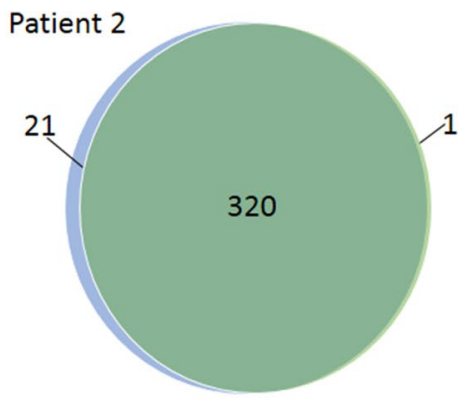

slightly lower for blood samples (median depth 107X-125X) than FFPE samples (median depth 134X-167X).

\section{Somatic variant detection}

Following variant filtration, the number of somatic variants (either SNVs or small indel) called per tumour using blood as a germline reference ranged from 11 to 341 and the number of variants called using normal breast tissue as a germline reference ranged from 12 to 321 . The overlap of somatic variants called on tumour versus blood and tumour versus normal breast tissue is shown in Fig. 1 and ranged from 76.9 to $93.6 \%$ (Table 2, S2). This indicates a good agreement between variants called using either germline reference. The specific somatic variants identified for each sample, and using both germline samples, are tabulated in the supplemental data. Somatic variants that are known recurrent driver mutations in breast cancer $[7,15,16]$ are also tabulated, for which agreement in calling using either germline remains very good (table S3, S4, S5).

Somatic variants identified using exome sequencing were validated using targeted sequencing consisting of an Ampliseq panel to test 139 selected variants (the specific variants tested are listed in the supplemental appendix, table S6). As outlined in the "Methods" section, variants with discrepant calls between each germline and variants of specific relevance to cancer were prioritised in this panel. $110 / 139(79.1 \%)$ of mutations were called using the

\section{Patient 5}
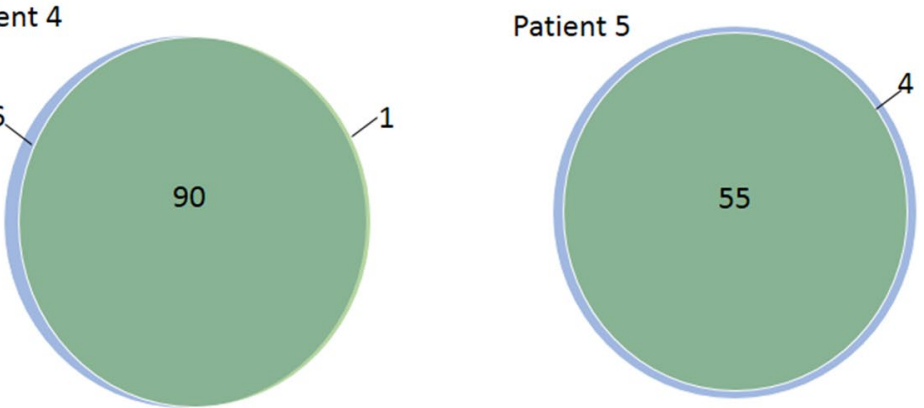

Somatic variants identified using normal breast tissue as germline reference

Fig. 1 The overlap of somatic variants called with blood or normal breast tissue as germline reference 
Table 2 The overlap of somatic variants (both SNVs and short indels) called in tumour using either blood or normal breast tissue as the germline reference using exome sequencing and targeted Ampliseq validation

\begin{tabular}{|c|c|c|c|c|}
\hline \multicolumn{5}{|c|}{ Exome sequencing } \\
\hline Sample no. & $\begin{array}{l}\text { Total variants versus } \\
\text { normal breast tissue }\end{array}$ & Total variants versus blood & Shared variants & $\begin{array}{l}\text { Percentage of variants shared using } \\
\text { normal breast or blood as reference }(\%)\end{array}$ \\
\hline 1 & 29 & 30 & 27 & 84.38 \\
\hline 2 & 321 & 341 & 320 & 93.57 \\
\hline 3 & 12 & 11 & 10 & 76.92 \\
\hline 4 & 85 & 90 & 84 & 92.31 \\
\hline 5 & 55 & 59 & 55 & 93.22 \\
\hline \multicolumn{5}{|c|}{ Targeted Ampliseq validation } \\
\hline Sample no. & $\begin{array}{l}\text { No of variants with } \\
\text { results }\end{array}$ & $\begin{array}{l}\text { Variant present in exome } \\
\text { sequencing using blood as } \\
\text { reference }\end{array}$ & $\begin{array}{l}\text { Variant present in exome sequenc- } \\
\text { ing using normal breast as refer- } \\
\text { ence }\end{array}$ & $\begin{array}{l}\text { Percentage of shared exome sequencing } \\
\text { variants validated by Ampliseq panel } \\
(\%)\end{array}$ \\
\hline 1 & 15 & 15 & 14 & 93.3 \\
\hline 2 & 57 & 57 & 41 & 71.9 \\
\hline 3 & 2 & 2 & 2 & 100.0 \\
\hline 4 & 28 & 28 & 27 & 96.4 \\
\hline 5 & 8 & 8 & 7 & 87.5 \\
\hline Total & 110 & 110 & 91 & \\
\hline
\end{tabular}

$\boldsymbol{S N} \boldsymbol{V}$ single nucleotide variation

Ampliseq panel. Of the 29 mutations that were not successfully called using the Ampliseq panel, but present in the exome sequencing data, 19 occurred at VAF less than $10 \%$ in exome sequencing and 11 of these had VAF less than $5 \%$. A further three mutations in the Ampliseq panel had lower than average coverage ( $<1000$ reads), and two mutations had poor mapping quality. This indicates a number of the mutations that were not successfully called had identifiable poor quality metrics.

Thirty nine of the 41 total somatic variants with a discrepant call between normal breast and blood were included in the Ampliseq panel. Of these, 35 variants were detected using blood and not normal breast, and four variants were detected using normal breast and not blood. Of the 39 discrepant variants in the Ampliseq panel, successful calls were obtained for 19 variants, all of which had been identified using blood as the germline, and not normal breast tissue. For the remaining 21 variants, there was no coverage in the relevant regions and hence it was not possible to discern mutation status. Manual review of the 19 variants suggested that for the majority of variants, the relevant base change only occurred in a small number of sequencing reads and often towards the end of reads (where read qualities decrease). In addition, the majority of changes were $\mathrm{G}$ to A and $\mathrm{C}$ to $\mathrm{T}$ base changes. Although it is not possible to definitively categorise these changes, the manual review indicates that both sequencing error and FFPE artefact explain a substantial proportion of these discrepancies.
Over 300 somatic variants were called with each reference germline from sample number 2 . This sample may have been a hypermutated tumour arising for example from mismatch repair deficiency or APOBEC-induced mutagenesis (see supplemental data for COSMIC somatic mutation signatures, figure S1). The high total number of somatic variants called in sample 2 means that it is not surprising that 15 of the 19 (78.9\%) variants with discrepant calls arose from this sample. It is possible that field cancerisation explains some of the discrepant calls in this sample; however, FFPEinduced artefact may have also increased the likelihood of discrepant calls.

\section{Matched analysis of CNA}

Estimates of tumour purity by a specialist histopathologist and the ASCAT analysis software package ranged from 52 to $90 \%$ (table S7). CNA detected using either matched blood or normal breast tissue as germline material are shown as copy number profiles and scatter plots in Fig. 2 for patients 1, 4 and 5, respectively, and for patients 2 and 3 in the supplementary appendix (figure S2). Visual comparison of these plots suggests considerable variation in genomic instability between samples but overall a good correlation between CNA identified using either germline reference. Pearson's correlation coefficient for paired comparisons of CNA using either reference ranged from 0.70 to 0.94 .

Validation of CNA was carried out using ddPCR for five genes. As explained above, these genes were selected as 

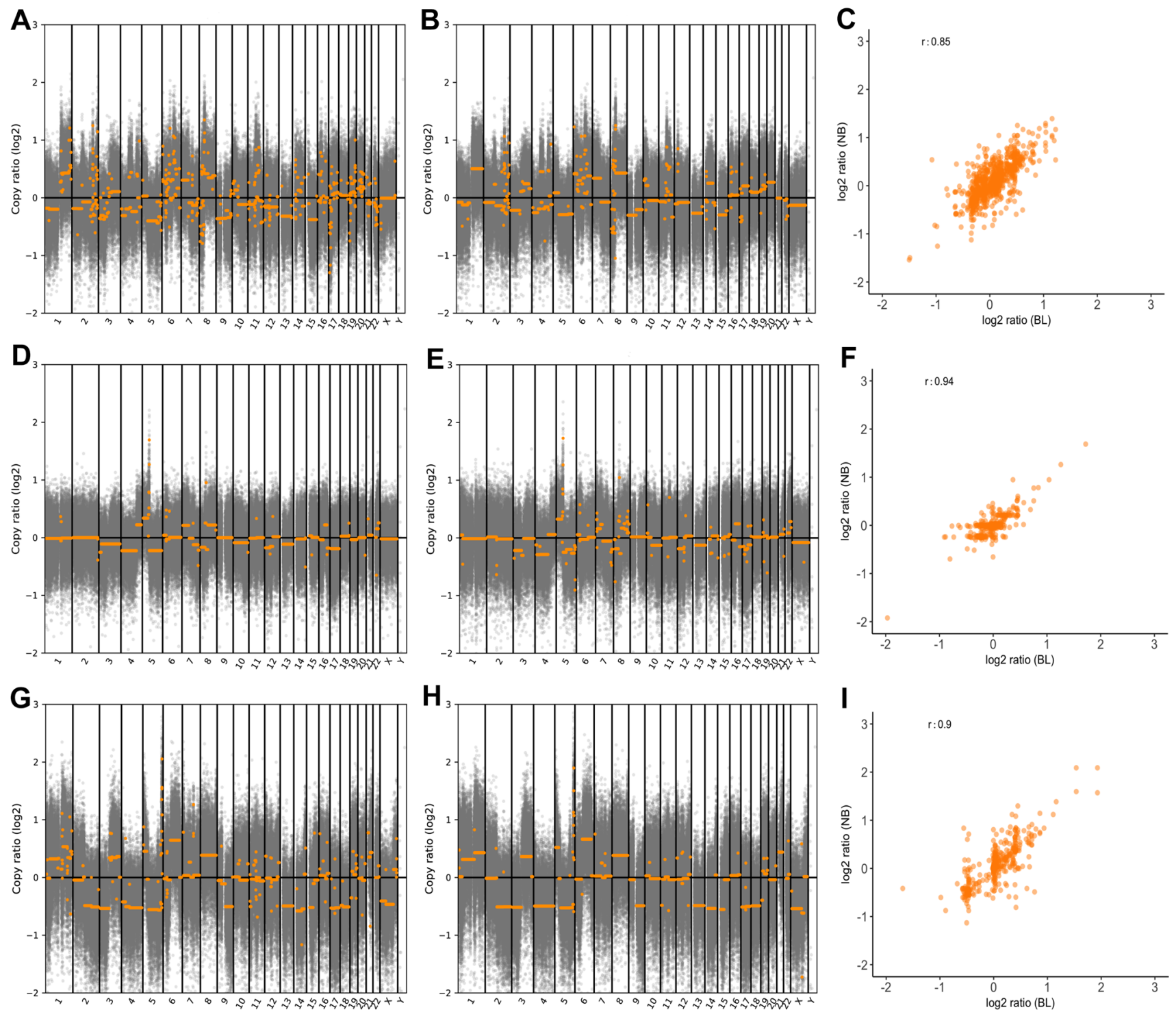

Fig. 2 Copy number profiles using normal breast or blood as germline reference and correlation between references. a and b Copy number profiles showing CNA called using both normal breast and blood as germline, respectively, for patient 1, c Scatterplot showing correlation of CNA using normal breast or blood as germline patient 1. $\mathbf{d}$ and e Copy number profiles showing CNA called using both normal breast and blood as germline respectively for patient 4 , f Scat- terplot showing correlation of CNA using normal breast or blood as germline patient 4. $\mathbf{g}$ and $\mathbf{h}$ Copy number profiles showing CNA called using both normal breast and blood as germline respectively for patient 5, i Scatterplot showing correlation of CNA using normal breast or blood as germline patient 5. CNA copy number alteration, $B L$ blood, $N B$ normal breast tissue

'pooled normal breast reference.' We considered this might be a useful approach to reduce sequencing noise, such as FFPE-induced artefact, from individual sample comparisons. These pooled references were then compared with individual tumour samples. Overall, these comparisons did not improve resolution of CNA and instead appeared to add artefactual noise. Copy number profiles using these pooled comparisons for patients 1 and 5 are shown in the supplementary appendix figure S3. A limitation of these 
Table 3 ddPCR validation of CNA

\begin{tabular}{llllll}
\hline Gene & Sample no. & \multicolumn{2}{l}{$\begin{array}{l}\text { Exome sequencing (absolute copy } \\
\text { number) }\end{array}$} & ddPCR ratio & ddPCR result \\
\cline { 3 - 5 } & & $\begin{array}{l}\text { Tumour versus } \\
\text { normal breast }\end{array}$ & $\begin{array}{l}\text { Tumour versus } \\
\text { blood }\end{array}$ & \\
\hline MALAT1 & 2 & 3 & 5 & 2.07 & Gain \\
IKBKB & 4 & 3 & 4 & 1.69 & Gain \\
TERT & 4 & 3 & 5 & 1.84 & Gain \\
HIST1H3B & 2 & 4 & 3 & 2.13 & Gain \\
IRF4 & 1 & 3 & 5 & 0.92 & No gain \\
\hline
\end{tabular}

$d d P C R$ droplet digital polymerase chain reaction pooled comparisons is that they were derived from only five patients.

\section{Discussion}

This study sought to identify if matched normal breast tissue from FFPE blocks, free of malignant or pre-malignant change on immunohistochemistry, could provide a reliable source of germline DNA with which tumour DNA could be compared using whole exome NGS. To help establish reliability, matched blood and tumour DNA were also compared. Findings from NGS were validated with targeted sequencing using an Ampliseq panel and droplet digital PCR. We acknowledge the small sample size which limits a rigorous assessment of the impact of factors such as specimen age on outcomes. However, this was a proof-of-principle study based on patients where all three of blood, normal tissue and tumour samples were available from a historical cohort.

We were able to prepare libraries and carry out whole exome sequencing using DNA from old archival FFPE samples retrieved from different treatment centres. This was despite the effects that fixation can have on DNA including hydrolysis, deamination and DNA-protein cross-linking, all of which can compromise library production. In addition, a good correlation between genomic aberrations called with blood as reference versus those called with normal tissue from the tumour resection specimen was seen.

With the use of stringent filtration criteria, there was a very good agreement between somatic variants identified, including for known recurrent driver mutations in breast cancer $[7,15,16]$, using either normal breast tissue or blood as a germline reference. Our filtration criteria were selected based on the MSK-IMPACT experience [12]. Whilst it is possible that less stringent criteria may have identified more true somatic variants (see supplementary data, table S3, S8), our concern is that more false positive variants, typically induced by FFPE artefact, would also have resulted. Overall, as expected, variants with a VAF of more than $10 \%$ were validated most consistently. Almost all variants with a discrepant call between each germline sample on exome sequence were included in the validation panel. Those discrepant calls that were successfully sequenced using the Ampliseq panel were validated as true somatic variants. It is difficult to confirm whether such discrepant calls using the different germline references relate to FFPE-induced artefact, sequencing error or field cancerisation, however, it is relevant that these discrepancies occurred infrequently and represented less than $10 \%$ of all variant calls.

It is encouraging that correlation in CNA using either reference germline was also very good across the five analysed samples. If possible, fresh frozen tissue is increasingly used for NGS, particularly whole genome or whole exome sequencing, because it avoids the artefacts associated with formalin fixation. Our results suggest it is reasonable to use FFPE tissue in this context. We recognise that a limitation of this study is that the ddPCR platform used for validation only enabled reliable assessment of copy number gains with four or more copies, and not losses, and that a fairly small number of genes were selected for validation.

We attempted a number of strategies to try and reduce noise from copy number data, including the use of pooled references and trying different segmentation and filtering approaches. Using the CBS segmentation method, along with the CNVkit "filter" option, removed a significant proportion of the spurious copy number segments; however, we did not see any significant improvement using the pooled reference approach. This could be due to the fact that our pooled comparison only used five samples. Most clinical studies with a pooled reference would involve a much larger pool of reference samples which is likely to improve reliability of CNA detection [12]. The use of online repositories of pooled reference germline DNA, e.g. from The Cancer Genome Atlas (TCGA) or International Cancer Genome Consortium (ICGC) databases could be an alternative source of germline genomic data.

The recently reported analysis by TCGA of DNA defects, epigenetics and gene expression in cancer-adjacent breast 
tissue [8] concluded that approximately $40 \%$ of benignappearing breast-adjacent tissue harboured genomic defects in DNA copy number, sequence, methylation status or in RNA sequence. However, for the 40 samples that underwent exome sequencing, clear detectable CNA were rare, occurring in $10 \%$ or less of cases. In addition, although $25 \%$ of cancer-adjacent samples had moderate to high levels of tumour-like somatic mutations, the variant allele fraction (VAF) was typically low. For example, five out of six mutations seen in TP53 occurred at a VAF of less than $1 \%$, all of these mutations would have been filtered by the stringent criteria typically used for FFPE samples and in this study. Whilst the field cancerisation changes characterised in detail in the TCGA report are important, we do not believe that they invalidate the use of FFPE normal breast DNA as a germline reference. It is important that careful histopathological review of normal breast tissue is carried out and appropriate filtering of somatic variants. We propose exclusion of somatic variants with genotype quality scores below 20 , alternative read count less than 10 , variant allele frequencies (VAFs) less than $2 \%$ or coverage less than 20 reads.

In summary, this study sought to establish if archival DNA extracted from histologically normal FFPE breast tissue blocks could be used as a germline reference against tumour DNA, when germline blood is not available. It is relevant that we used old archival tissue samples retrieved from different treatment centres where they had been stored for at least 14 years. Somatic variants identified using either matched blood or normal breast tissue had a very good agreement, suggesting that normal breast tissue can be used as a reliable surrogate to identify these aberrations with appropriate filtration criteria. Such filtration criteria appear to minimise the impact of both FFPE-induced artefact and any field cancerisation changes that may be present. Although CNA were more complicated to directly compare, copy number abnormality calls were consistent using either germline reference and key copy number gains were validated. Overall, this study suggests that normal breast DNA from archival FFPE blocks lacking malignant or pre-malignant change can be used as a surrogate germline and offers a robust alternative to use of DNA from blood for whole exome sequencing.

Acknowledgements We acknowledge funding to the NIHR Biomedical Research Centre at The Royal Marsden NHS Foundation Trust and The Institute of Cancer Research, London. We would like to thank Ioannis Assiotis and Penny Robinson from the ICR Tumour Profiling Unit for their help with sample preparation and exome sequencing and Amanda Swain for advice with the sequencing strategy. We would also like to thank Ben O'Leary and Ros Cutts for advice with validation of exome sequencing, Selva Anbalagan for help creating cell pellets and Irene Chong for provision of cell line controls. We are grateful to Nick Turner's laboratory for technical assistance with droplet digital PCR. AW is supported by a Cancer Research UK Clinical Research Fellowship. We thank all those involved with the START trials. Funding for the
START trials (CRUK/96/001) was provided by Cancer Research UK, the UK Medical Research Council (MRC 080A A4307), and the UK Department of Health (Grant G9600656). Continued data collection and analysis is made possible by a core grant from Cancer Research UK to the ICR-CTSU. The tumour collection for Trans-Fraction (RMH CCR3079) was supported by The Royal Marsden Hospital Charity (CP Ref 09103). AW is funded by a Cancer Research UK Clinical Research Fellowship. NS is funded by an ICR Clinician Scientist Fellowship Award.

\section{Compliance with Ethical Standards}

Conflict of interest The authors declare that they have no conflict of interest.

Ethics approval Trans-Fraction was approved by The Royal Marsden Research Ethics Committee (08/H0801/53) on 23 July 2008. The START trial was approved by South East London Multicentre Research Ethics Committee (MREC 98/96) on 30 September 1998 and all patients provided informed consent including use of tissue and blood for research purposes.

Open Access This article is distributed under the terms of the Creative Commons Attribution 4.0 International License (http://creativeco mmons.org/licenses/by/4.0/), which permits unrestricted use, distribution, and reproduction in any medium, provided you give appropriate credit to the original author(s) and the source, provide a link to the Creative Commons license, and indicate if changes were made.

\section{References}

1. Heaphy CM, Griffith JK, Bisoffi M (2009) Mammary field cancerization: molecular evidence and clinical importance. Breast Cancer Res Treat 118(2):229-239

2. Larson PS, Schlechter BL, de las Morenas A, Garber JE, Cupples LA, Rosenberg CL (2005) Allele imbalance, or loss of heterozygosity, in normal breast epithelium of sporadic breast cancer cases and BRCA1 gene mutation carriers is increased compared with reduction mammoplasty tissues. J Clin Oncol 23(34):8613-8619

3. Botti C, Pescatore B, Mottolese M, Sciarretta F, Greco C, Di Filippo F, Gandolfo GM, Cavaliere F, Bovani R, Varanese A et al (2000) Incidence of chromosomes 1 and 17 aneusomy in breast cancer and adjacent tissue: an interphase cytogenetic study. J Am Coll Surg 190(5):530-539

4. Meeker AK, Hicks JL, Gabrielson E, Strauss WM, De Marzo AM, Argani P (2004) Telomere shortening occurs in subsets of normal breast epithelium as well as in situ and invasive carcinoma. Am J Pathol 164(3):925-935

5. Heaphy CM, Bisoffi M, Fordyce CA, Haaland CM, Hines WC, Joste NE, Griffith JK (2006) Telomere DNA content and allelic imbalance demonstrate field cancerization in histologically normal tissue adjacent to breast tumors. Int J Cancer J 119(1):108-116

6. Xie B, Freudenheim JL, Cummings SS, Singh B, He H, McCann SE, Moysich KB, Shields PG (2006) Accurate genotyping from paraffin-embedded normal tissue adjacent to breast cancer. Carcinogenesis 27(2):307-310

7. Nik-Zainal S, Davies H, Staaf J, Ramakrishna M, Glodzik D, Zou X, Martincorena I, Alexandrov LB, Martin S, Wedge DC et al (2016) Landscape of somatic mutations in 560 breast cancer whole-genome sequences. Nature 534(7605):47-54 
8. Troester MHK, D'Arcy M, Cherniack A, Stewart C, Koboldt D, Gordon Robertson AMA et al (2016) DNA defects, epigenetics, and gene expression in cancer-adjacent breast: a study from The Cancer Genome Atlas. Breast Cancer 2:16007 (published online 4 May 2016).

9. Cingolani P, Platts A, Wang le L, Coon M, Nguyen T, Wang L, Land SJ, Lu X, Ruden DM (2012) A program for annotating and predicting the effects of single nucleotide polymorphisms, SnpEff: SNPs in the genome of Drosophila melanogaster strain w1118; iso-2; iso-3. Fly (Austin) 6(2):80-92

10. Talevich E, Shain AH, Botton T, Bastian BC (2014) CNVkit: Genome-wide copy number detection and visualization from targeted DNA sequencing. PLOS Comput Biol 12(4):e1004873

11. Olshen AB, Venkatraman ES, Lucito R, Wigler M (2004) Circular binary segmentation for the analysis of array-based DNA copy number data. Biostatistics 5(4):557-572

12. Cheng DT, Mitchell TN, Zehir A, Shah RH, Benayed R, Syed A, Chandramohan R, Liu ZY, Won HH, Scott SN et al (2015) Memorial sloan kettering-integrated mutation profiling of actionable cancer targets (MSK-IMPACT): a hybridization capture-based next-generation sequencing clinical assay for solid tumor molecular oncology. J Mol Diagn 17(3):251-264

13. Weaver S, Dube S, Mir A, Qin J, Sun G, Ramakrishnan R, Jones RC, Livak KJ (2010) Taking qPCR to a higher level: Analysis of CNV reveals the power of high throughput qPCR to enhance quantitative resolution. Methods 50(4):271-276

14. Hindson BJ, Ness KD, Masquelier DA, Belgrader P, Heredia NJ, Makarewicz AJ, Bright IJ, Lucero MY, Hiddessen AL, Legler TC et al (2011) High-throughput droplet digital PCR system for absolute quantitation of DNA copy number. Anal Chem 83(22):8604-8610

15. Pereira B, Chin SF, Rueda OM, Vollan HK, Provenzano E, Bardwell HA, Pugh M, Jones L, Russell R, Sammut SJ et al (2016) The somatic mutation profiles of 2,433 breast cancers refines their genomic and transcriptomic landscapes. Nat Commun 7:11479

16. Cancer Genome Atlas N (2012) Comprehensive molecular portraits of human breast tumours. Nature 490(7418):61-70 